\title{
Performance of Serial Underactuated Mechanisms: Number of Degrees of Freedom and Actuators
}

\author{
Ravi Balasubramanian and Aaron M. Dollar
}

\begin{abstract}
While underactuated mechanisms have become popular in robot-hand designs because of their passive adaptability, existing systems utilize only one actuator to produce motion in the multiple degrees of freedom in the serial chain of each finger. In this paper, we explore how the performance of an underactuated serial link chain changes as more actuators are added. The fundamental question of what extra capability an additional actuator provides to an underactuated system and how best to implement it has not yet been quantified in the literature. Using a simple linear underactuated mechanism, we show that the performance of a singleactuator system (measured as the average number of contacts made with the environment) quickly plateaus as the number of degrees of freedom of the mechanism is increased. Also, we show that as the number of actuators is increased, the system's passive adaptability improves as the mechanism implementation spreads the actuators across the joints.
\end{abstract}

\section{INTRODUCTION}

There are two primary approaches in current robotic design to specifying how a robot is actuated. The traditional approach has been to use an actuator for each degree of freedom to produce a fully-actuated system. However, this approach often results in bulky designs requiring complex control algorithms and elaborate sensing modalities for each control input. Recently, underactuated mechanisms that have fewer actuators than degrees of freedom have become popular in robot hand research, since the underactuated mechanism's unconstrained freedoms allow it to adapt to environmental constraints without any sensing [1], [2], [3], [4], [5], [6]. However, most current underactuated hand designs use just one actuator to actuate the many degrees of freedom in a robotic hand. This paper explores how the performance of an underactuated serial link chain changes as more actuators are utilized. We also explore how the routing mechanism used to transmit

R. Balasubramanian and A. M. Dollar are with the Department of Mechanical Engineering at Yale University, New Haven, CT. \{ravi.balasubramanian, aaron.dollar\}eyale.edu actuation to the degrees of freedom influences the system's adaptability.

A key performance goal of underactuated robotic hands is to produce power grasps and passively make contact at multiple links, thereby providing the robot the ability to apply forces on the object from multiple contact points and potentially improving grasp stability. This is achieved by routing the force from the single actuator to two [1], [7], three [2], [8], [9], [10], and even eleven [3] degrees of freedom in a finger through cabledriven mechanisms or linkage mechanisms (see Fig. 1). These mechanisms permit the distal link to move even after the proximal link makes contact with an object, a property commonly referred to as adaptability [1], [2], [11]. Note that as these fingers are incorporated into a robot hand, these same adaptive mechanisms are used to actuate multiple fingers in parallel as well to provide adaptability between fingers [12], [8], [1]. However, this paper focuses only on the adaptability of a serial underactuated chain.

The limited number of actuators and the uncertainty in object location and shape can lead to undesirable situations in which not all links of the chain make contact with the object. Specifically, a decreased number of contacts results in reduced grasp strength and a reduced ability to resist disturbance forces. Furthermore, unconstrained degrees of freedom (that is, links without contact constraints) permit the hand to reconfigure in response to an external disturbance or internal actuation, which may result in a weakened grasp [13].

In this paper, we explore two specific problems relating to underactuated mechanisms: 1) Given a singleactuator system, how does the system's adaptability vary as the number of degrees of freedom increases? 2) Given a $n$ degree-of-freedom system, how does the performance vary with an increasing number of actuators, and how should those actuators be best routed to the degrees of freedom? Motivated by the robotic grasping problem, for simplicity we will use the number of contacts the system makes with the object on 


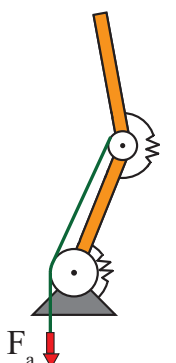

(a)

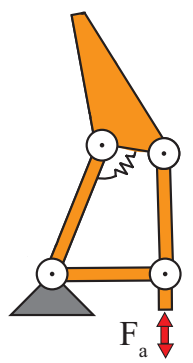

(b)
Fig. 1. A schematic of the common underactuated mechanisms used in robot hand designs: a) a cable-driven mechanism (note that the proximal joint is a free-spinning pulley) and b) a linkage-driven mechanism.

completing the grasping process as the primary performance metric (even though other metrics such as force application capability are important as well). Since the grasping problem in unstructured environments has tremendous uncertainty, we use a probabilistic analysis to quantify system performance.

The majority of underactuated mechanisms described in the robot hand literature utilize revolute degrees of freedom. However, the non-linearity of their kinematics and the variety of contact modes (such as sliding and rolling contact) reduce the generality of the analysis and substantially increase the already large parameter space, which includes joint stiffnesses, transmission mechanisms, and object shapes. To minimize the influence of these factors, our analysis will use a linear underactuated mechanism that we first introduced in [11] (see Fig. 2). The simple geometry of the linear underactuated mechanism's degrees of freedom and contact modes helps retain focus on how the number of actuators influences system performance.

\section{THE LINEAR UNDERACTUATED MECHANISM}

The linear underactuated mechanism studied in this paper operates in a single dimension. Fig. 2 shows a $n=3$ degree of freedom system, where each degree of freedom is a compliant prismatic joint with unit joint travel. For this system to be underactuated, the $n=3$ degrees of freedom can be actuated by up to $n-1$ actuators (that is, the number of actuators $m \in\{1,2\}$ ). Each actuator has some bounded force capability, and the force from the actuator(s) can be transmitted to the degrees of freedom through a variety of transmission mechanisms, which will be explored in section II-A. Note that the force provided by the single actuator to the distal joint in the mechanism in Fig. 2a causes the

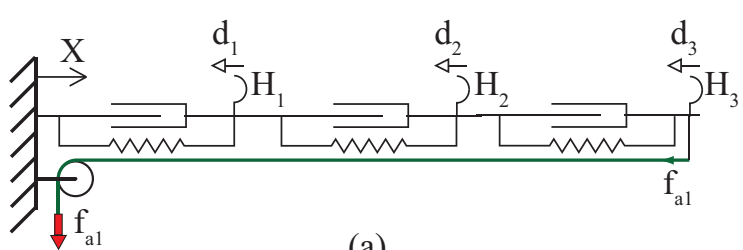

(a)

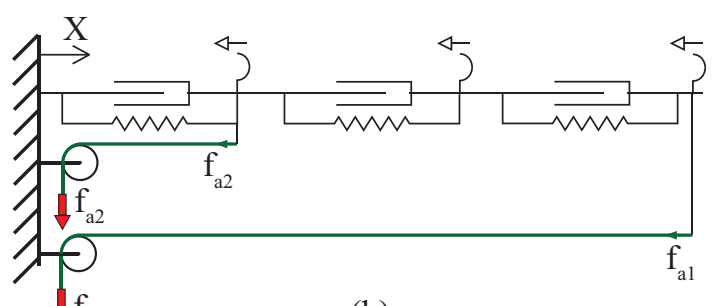

(b)

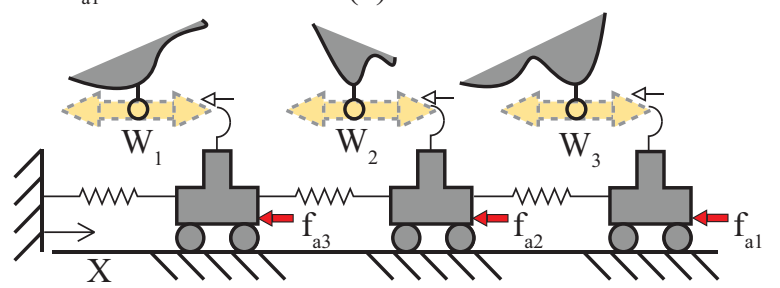

(c)

Fig. 2. A three degree-of-freedom linear underactuated system driven by a cable-driven mechanism which routes forces from a) a single actuator (inserted at distal joint) and b) two actuators (inserted at proximal and distal joint). c) A schematic drawing of a linear underactuated system with generalized joint actuation interacting with the environment. The white-headed arrows represent joint travel, the solid (red) arrows forces, and the thick dotted arrows uncertainty in object prong location.

proximal and middle joints also to compress in the absence of any contact since the mechanism is linear. Thus, the net displacement of the distal link in free space is three times the displacement of the proximal link.

Each degree of freedom has a "hook" $H_{i}, i=1, \ldots, n$, through which it can make contact with the environment, which also has hooks $W_{i}, i=1, \ldots, n$. We assume that there exists a hook $W_{i}$ for each degree of freedom, but its location is not known a priori (Fig. 2c shows the uncertainty in prong location using the thick dotted arrows). Such contacts place constraints on the mechanism's motion. Specifically, if the distal link of the mechanism in Fig. 2a made contact, then the system is locked since the actuator cannot apply any forces on the proximal and middle joints.

With more than one actuator, there exists significant choice in how the actuators can be utilized. For example, the system in Fig. $2 b$ has two actuators, and the system is not locked when the distal link makes contact, since the second actuator can still produce motion in the 
proximal and middle joints. Note also that the second actuator only causes the distal and middle joints to translate (and not compress) when the mechanism is actuated in free space. Thus, in contrast to the effect of the first actuator, the net displacement of the distal link in free space due to the second actuator is equal to the displacement of the proximal link. Such differences in the capability of actuators inserted at different points in the serial chain have implications in the grasping process, since joint travel is critical for making contact with the environment. These effects will be discussed in the following sections. Also, the various control policies available to a multi-actuator system will be explored in section II-B.

\section{A. Transmission Mechanisms}

The transmission mechanism determines the magnitude of forces the actuator can apply at a specific degree of freedom. While the examples shown in Fig. 2 use cable-driven mechanisms, our analysis applies to the use of any actuating mechanism such as linkages or pneumatics. From here on, a joint force will be represented by a force proportional to the actuator force $f_{a j}, j=1 \ldots m$, which is applied at the joint without explicitly specifying how that force was created. We assume for simplicity that the actuators can only pull, and not push.

The transmission mechanism can be represented as an actuator Jacobian $J_{a} \in \mathbb{R}^{m \times n}$ such that the joint forces $\tau$ can be computed as $\tau=J_{a}^{T} f_{a}$, where $f_{a}=$ $\left[\begin{array}{lll}f_{a 1} & \ldots & f_{a m}\end{array}\right]^{T} \in \mathbb{R}^{m}$. For the single-actuator mechanism in Fig. $2 \mathrm{a}, J_{a}=\left[\begin{array}{lll}1 & 1 & 1\end{array}\right]$. For simplicity, we assume that the first actuator is always inserted at the distal joint.

For the mechanism in Fig. $2 b, J_{a}=\left[\begin{array}{lll}1 & 1 & 1 \\ 1 & 0 & 0\end{array}\right]$, where the second row corresponds to the second actuator which is inserted at the proximal joint. Note that the second actuator could have been inserted at a more distal degree of freedom as well (up until the second degree of freedom) resulting in a different actuator Jacobian. Thus, with more than one actuator, there are several transmission mechanisms to choose from. We assume that the two actuators are not routed to the same joint and the higher number actuators are always inserted more proximally.

Also, one can design transmission mechanisms where a particular actuator is routed multiple times to various joints, producing actuator Jacobians that have elements larger than unity (see [11] for examples). In
TABLE I

Number of POSSIBLE TRANSMISSION MECHANISMS AND Control Policies With Six Degrees of Freedom

\begin{tabular}{c|c|c}
\hline $\begin{array}{c}\text { Number of } \\
\text { actuators }(\mathrm{m})\end{array}$ & $\begin{array}{c}\text { Possible transmission } \\
\text { mechanisms }\end{array}$ & $\begin{array}{c}\text { Possible control } \\
\text { policies* }\end{array}$ \\
\hline 1 & 1 & 1 \\
2 & 5 & 3 \\
3 & 10 & 13 \\
4 & 10 & 75 \\
5 & 5 & 541 \\
\hline \hline
\end{tabular}

*for each mechanism

this paper, we will not consider such mechanisms for simplicity. Thus, the number of possible transmission mechanisms with $m$ actuators and $n$ degrees of freedom is equal to the binomial coefficient $\left(\begin{array}{c}n-1 \\ m-1\end{array}\right)$ (see Table I).

\section{B. Control Policies}

With more than one actuator, there are numerous ways in which the actuators can be utilized in the grasping process, each method called a control policy. Each control policy potentially leads to different performance.

One control policy is to use all the actuators simultaneously to produce motion, that is $c p_{1}:=$ $\left\{\left(f_{a 1}, f_{a 2}, \ldots, f_{a m}\right)\right\}$, where the brackets indicate that all the actuators are used simultaneously. If a particular actuator does not produce any more motion because the joint it is inserted at has made contact, then the other actuators continue to produce motion until all actuators do not produce any motion.

Another control policy $c p_{2}:=\left\{\left(f_{a 1}\right),\left(f_{a 2}\right), \ldots\left(f_{a m}\right)\right\}$ is to use each actuator individually starting from the most distal actuator and then moving to the most proximal actuator. Here the brackets indicate that only one actuator is used at a time. The switch from one actuator to another occurs only when an actuator produces no further motion (when the joint the actuator is inserted at has made contact) or if that actuator has no remaining force capability to produce motion (the actuator's force being consumed by the joint stiffnesses). Note that when a joint, whose motion is controlled by an actuator, makes contact with the object, only the remaining actuation force is available to produce motion at the other joints that the actuator controls. For example, if the mechanism in Fig. 2a utilized $25 \%$ of its actuation force to make contact with the proximal link, only $75 \%$ of its actuation force remains to produce motion in the other joints. An alternate 
control policy $c p_{2}:=\left\{\left(f_{a m}\right), \ldots,\left(f_{a 1}\right)\right\}$ is to the reverse the order of actuator use and start from an actuator that is inserted most proximally and move sequentially to more distal actuators.

Furthermore, the control policies $c p_{1}, c p_{2}$, and $c p_{3}$ can be used recursively with each subset of actuators. Specifically, a control policy $c p_{4}:=$ $\left\{\left(f_{a 1}\right),\left(f_{a 2}, \cdots f_{a m}\right)\right\}$ can use the first actuator individually and then use the remaining actuators simultaneously. Indeed, the number $T_{c p}(m)$ of possible control policies with $m$ actuators grows exponentially as

$$
T_{c p}(m)=1+\sum_{i=1}^{m-1}\left(\begin{array}{c}
m \\
i
\end{array}\right) T_{c p}(m-i),
$$

with each control policy producing potentially different behaviors and $T_{c p}(1)=1$ (see Table I).

\section{Grasping Process}

Given an underactuated system with a defined transmission mechanism and a control policy, the grasping process involves executing the control policy completely until all joints can no longer move. With more degrees of freedom, actuators, and a step-by-step control policy such as $c p_{2}$ (see section II-B), the grasping process can occur in multiple stages. Specifically, with $c p_{2}$ and the mechanism shown in Fig. $2 \mathrm{~b}$, actuator 1 will cause all the joints to compress. If the distal joint first makes contact, then actuator 1 produces no more motion. Then the next actuator in the control policy, actuator 2, is utilized. This causes the proximal joint to compress, produce translation of joint 2 and extension of the springs in joints 2 and 3 until the next contact. If joint 1 makes contact, then the grasping process is complete since there are no more actuators to produce motion. If on the other hand, joint $2 \mathrm{had}$ made contact, then the mechanism can still reconfigure and the grasping process continues until all the joints are locked. Such a grasp is called a power grasp and is a key goal of grasping with underactuated mechanisms.

\section{Evaluating Grasping Performance}

There are several heuristics used in the robotic grasping literature to measure grasp quality [14], [15], but in general the goal of the grasping process is to maximize the number of contacts between the fingers and the object. Each contact would enable the finger to apply an additional force to the object and resist external disturbances.

To model the uncertainty in the grasping process, where the object's exact location and shape (modeled by prong location) are unknown, we simulated many object possibilities in order to quantify a system's average performance on a generic object. Thus, the object prong locations were randomized over the unit joint travel distance (indicated by the thick dotted arrows in Fig. 2c), and each underactuated system attempted to make contact with all the world prongs using the grasping process delineated in section II-C. The number of contacts that the system made with each randomized object was recorded. For each underactuated system, we compute the probability of occurrence of each contact mode after the grasping process for each randomized object. Then, for each underactuated system, we can compute the average number of contacts expected for a generic object. A system with a greater average number of contacts is rated higher.

\section{E. Static Analysis}

The linear underactuated mechanism's joint-travel and force-application capabilities during the grasping process can be computed using a statics analysis at each joint. The static balance at each contact state is given by

$$
J_{a}^{T} f_{a}+K d_{i}+J_{c}^{T} f_{i}=0,
$$

where $K \in R^{3 \times 3}$ represents mechanism's stiffness, $J_{c} \in$ $R^{3 \times 3}$ the contact-constraint Jacobian, $f_{i}$ the contact force at joint $i$, and $d_{i}$ the joint travel. In this paper, we assume that the mechanism's stiffness $K$ is an identity matrix even though it is a tunable parameter.

Note that the static equations (2) need to be solved in conjunction with the relevant contact constraints:

$$
\begin{array}{lc}
\text { Proximal contact } & d_{1}=0, \\
\text { Middle contact: } & d_{1}+d_{2}=0,
\end{array}
$$$$
\text { Distal contact: } \quad d_{1}+d_{2}+d_{3}=0 \text {. }
$$

In this paper, the primary focus is on the mechanism's contact state at the end of grasping process and not the intermediate contact states or force application capabilities (as was explored in [11]).

\section{RESULTS}

Using the linear underactuated system presented in section II, we explored how the grasping performance of a single-actuator system varied as the number of degrees of freedom increased from $n=2$ to $n=6$. We also explored how the grasping performance of a $n=6$ degree of freedom system varies as it is actuated by a differing number of actuators (from $m=1$ to $m=$ 5), different mechanism implementations, and different control policies. The location of each world prong was randomly sampled from a uniform distribution over 
TABLE II

Single-Actuator System: Performance VARIATION With DEGREES OF FREEDOM

\begin{tabular}{c|c}
\hline $\begin{array}{c}\text { Number of degrees } \\
\text { of Freedom }(\mathrm{n})\end{array}$ & $\begin{array}{c}\text { Expected number of } \\
\text { contacts* }\end{array}$ \\
\hline 2 & 1.2 \\
3 & 1.5 \\
4 & 1.6 \\
5 & 1.8 \\
6 & 1.9 \\
\hline \hline
\end{tabular}

*Standard error is less than 0.04 in estimates.

the unit length of the degree of freedom (five hundred samples), and system performance was averaged across all the instances.

We assumed that all the actuators begin with two units of force, where a single unit of force is sufficient to produce joint motion equal to the length of the joint while overcoming the unit joint stiffness. We verified that at the end of the grasping process, all the joints could no longer move and all the actuators had some remaining actuation force capability, indicating that the mechanism was constrained by external contacts.

Interestingly, we noticed that the different control policies (available for a transmission mechanism with more than one actuator) produced the same number of contacts after the grasping process. Thus, the control policies did not make a difference in the final contact state of the system. The rest of the results section will focus only on how the number of actuators and the transmission mechanism influence system performance.

\section{A. The Single-Actuator System: Performance Variation With Increasing Degrees of Freedom}

Fig. 3 shows the variation in performance of a singleactuator system as the number of degrees of freedom increases from $n=2$ to $n=6$. We notice that with two and three degrees of freedom, a single contact is the most likely contact mode. However, for four and higher degrees of freedom, the double-contact mode has highest likelihood. A weighted average of these results indicate that the expected average number of contacts marginally increases as the number of degrees of freedom increases (see Table II).

\section{B. Fixed Degree-Of-Freedom System: Variation With Number of Actuators and Transmission Mechanism}

Fig. 4 shows how the performance of a six degree of freedom system varies as the number of actuators that

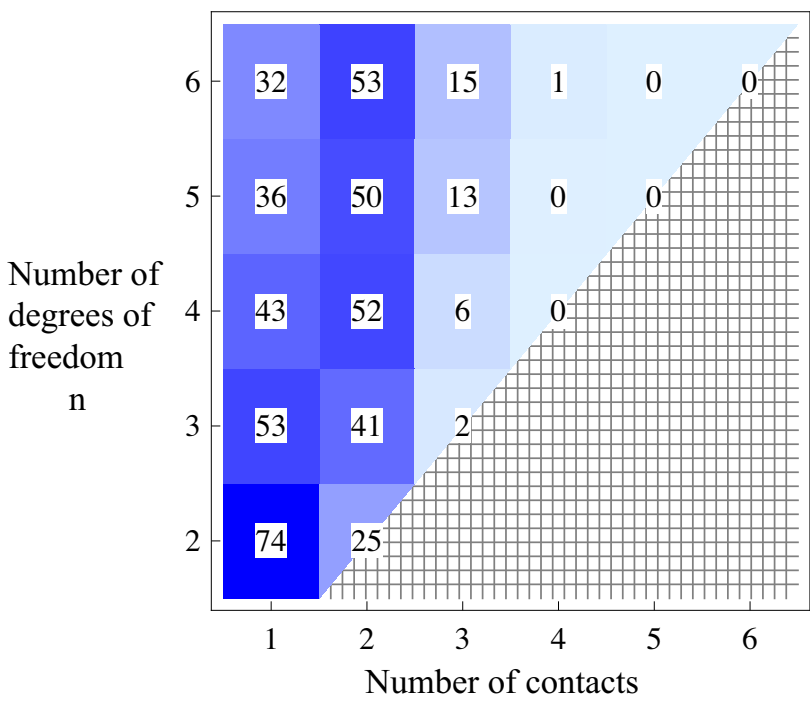

Fig. 3. Expected number of contacts for a single-actuator linear underactuated system with varying degrees of freedom. The numbers in the grid represent the probability (percentage, error in estimates less than $0.8 \%$ in all cases) of occurrence of the particular contact mode. Events in the dense checked region are not possible.

control it are increased from $m=1$ to $m=5$ and different transmission mechanisms are used. As expected, we notice that the expected number of contacts increases with more actuators. However, the transmission system influences performance as well. Specifically, if the actuators are inserted most distally, the expected number of contacts is significantly smaller than the expected number of contacts if the actuators are inserted most proximally. However, for the $m=3$ and $m=4$ actuator case, there is some variability in system performance as the insertion point moves distally, and this is discussed in section IV-B.

C. Fixed Degree-Of-Freedom System: Best Performance Variation With Increasing Number of Actuators

Fig. 5 shows how the best performance (across all possible transmission mechanisms) of a six degree of freedom system changes as the number of actuators are increased from $m=1$ to $m=5$. We notice that the most likely contact mode is $m+1$ contacts, except for the five actuator case where the most likely number of contacts is five. Again, these results can be averaged to compute the expected number of contacts with a generic object as the number of actuators increase (see Table III).

\section{DISCUSSION}

\section{A. Limitations of Single-Actuator Systems}

From the analysis of the linear underactuated system, we notice that each additional degree of freedom pro- 


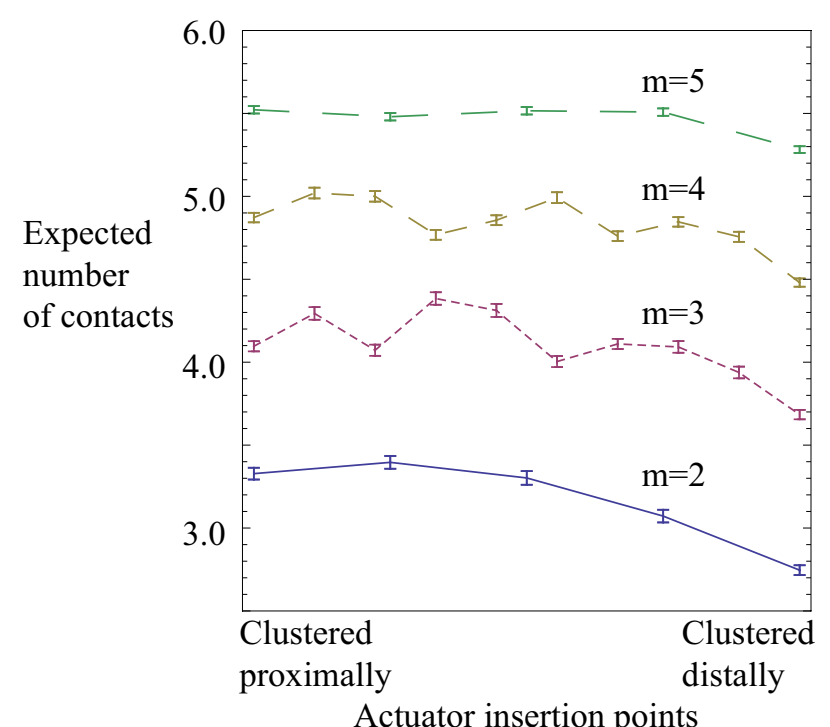

Fig. 4. Expected number of contacts for a $n=6$ degree-of-freedom linear underactuated system as the number of actuators is increased from $m=2$ to $m=5$. The $\mathrm{X}$-axis represents mechanism routing where the insertion points become more distal.

TABLE III

FiXed Degree-Of-Freedom System: Best Performance VARIATION WITH NUMBER OF ACTUATORS

\begin{tabular}{c|c}
\hline $\begin{array}{c}\text { Number of } \\
\text { actuators }(\mathrm{m})\end{array}$ & $\begin{array}{c}\text { Expected number of } \\
\text { contacts* }\end{array}$ \\
\hline 1 & 1.9 \\
2 & 3.2 \\
3 & 4.1 \\
4 & 4.8 \\
5 & 5.4 \\
\hline \hline
\end{tabular}

*Standard error is less than 0.02 in estimates.

vides only marginal advantage in a the single-actuator system (see Table II and Fig. 3). A similar analysis is also required for the planar underactuated systems prevalent in robot hand research. While prior research has shown that power grasps with multiple contacts between the finger and the object are possible (by carefully designing joint compliances) even when the single actuator controls many degrees of freedom, they include assumptions about hand placement (for example, object pushed against the hand's palm) and object shape (for example, spherical shape). The expected performance of these robot hands in terms of the number of contacts the mechanism makes on average across all possible objects is still unclear. To our knowledge, this is the first paper that explores the variation in expected number of contacts for a single actuator serial chain

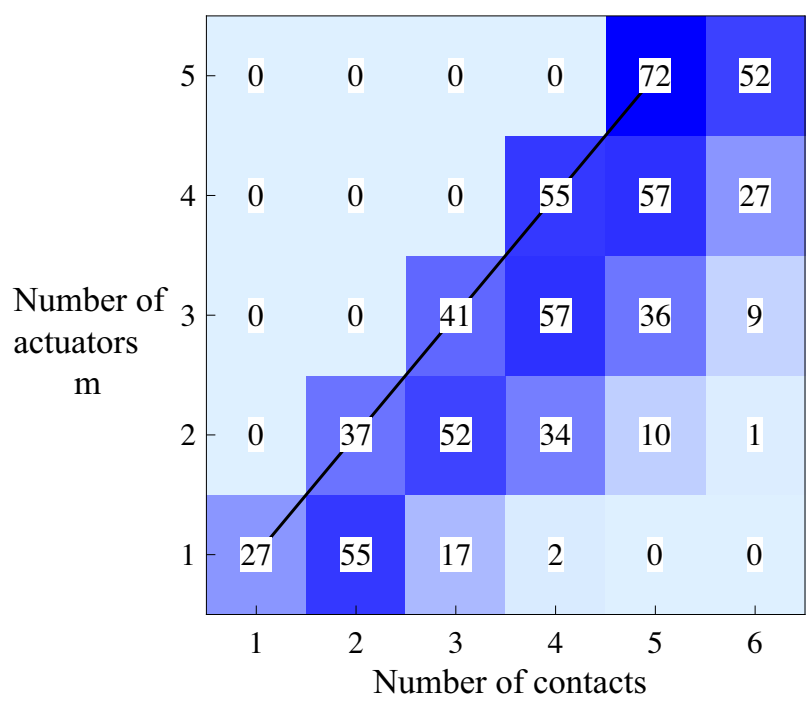

Fig. 5. Best-case expected number of contacts (across all transmission mechanisms) for a $n=6$ degree-of-freedom linear underactuated system as the number of actuators is increased from $m=1$ to $m=5$. The numbers in the grid represent probability (percentage, error in estimates less than $0.42 \%$ in all cases) of occurrence of the particular contact mode. The black solid line represents the line where number of contacts equals number of actuators.

with increasing degrees of freedom.

We would also like to point out a key difference between the linear underactuated system and the planar underactuated systems that utilize revolute joints. Since the linear underactuated system has only one dimension, a contact on one joint can nullify the actuation force on another joint. In contrast, actuation forces can still be transferred to the revolute joints after the planar systems make contact with the environment because of the rotational joint kinematics. This depends on the specific contact modes (rolling versus sliding) which will determine the mechanism's ability to reconfigure [13]. Thus, these factors that influence the mechanism's adaptability must be kept in mind when analyzing the performance of planar underactuated systems.

\section{B. Multi-Actuator Systems}

As expected, a system with more actuators has greater adaptability irrespective of how the actuation is routed (see Fig. 4). However, the control policy, or the sequence of actuator utilization, does not make a difference in terms of the number of contacts the mechanism makes with the object after the grasping process. This is a surprising and useful result which indicates that even with multiple actuators, the robotic hand can use the actuators in any order to produce an identical final contact mode. However, it was still 
useful to explore the various control policies in section II-B to show the completeness of our methods. However, the contact sequence does indeed depend on the sequence of actuator use. If the contact sequence is important (for example, making contact sequentially from the proximal link to the distal link to ensure an enveloping grasp), then the robot must plan the sequence of actuator use as well.

In terms of mechanism routing, we notice that a system where the actuator insertion points are spread across the serial chain improve mechanism adaptability. This is particularly noticed in the poor performance of the systems where all the actuators are inserted most distally. In these cases, contacts at the distal joints would completely lock the system. Interestingly, with $m=3$ and $m=4$ actuators, as the routing becomes more distal there is some variability in performance. This is because of the poor performance of mechanisms where the insertion points of the higher-order actuators are adjacent when compared with mechanisms where the insertion points of the higher-order actuators are spread apart. We also notice that the best performance with $m$ actuators increases linearly until saturating at $n-1$ actuators. Thus, there is a linear advantage with each additional actuator. Future work includes an analysis of a multi-actuator system's ability to individually control the contact forces and permit object manipulation.

\section{REFERENCES}

[1] A. M. Dollar and R. D. Howe. The SDM Hand: A highly adaptive compliant grasper for unstructured environments. Internat. J. Robotics Res, 2010.

[2] L. Birglen, T. Laliberté, and C. Gosselin. Underactuated Robotic Hands. Springer, 2008.

[3] S. Hirose and Y. Umetani. The development of soft gripper for the versatile robot hand. Mechanism and IVlechine Theory, 13:351-359, 1978.

[4] A. Rovetta. On functionality of a new mechanical hand. ASME J. Mechanical Design, 103:277-280, 1981.

[5] M. Rakic. Multifingered hand with self-adaptability. Robotics and Computer-Integrated Manufact., 3(2):269-276, 1989.

[6] R. M. Crowder, V. N. Dubey, P. H. Chappell, and D. R. Whatley. A multi-fingered end effector for unstructured environments. In Proc. IEEE Internat. Conf. on Robotics and Automation, pages 3038-3043, 1999.

[7] M. Kaneko, M. Higashimori, R. Takenaka, A. Namiki, and M. Ishikawa. The $100 \mathrm{G}$ capturing robot too fast to see. ASME/IEEE Trans. on Mechatronics, 8(1):37-44, 2003.

[8] Y. Kamikawa and T. Maeno. Underactuated five-finger prosthetic hand inspired by grasping force distribution of humans. In Proc. IEEE/RSJ Internat. Conf. on Intell. Robots and Sys., pages 717-722, 2008.
[9] M. C. Carrozza, C. Suppo, F. Sebastiani, B. MASSA, F. Vecchi, R. Lazzarini, M. R. Cutkosky, and P. Dario. The spring hand: Development of a self-adaptive prosthesis for restoring natural grasping. Autonomous Robots, pages 125-141, 2004.

[10] J. D. Crisman, C. Kanojia, and I. Zeid. Graspar: A flexible, easily controllable robotic hand. IEEE Robotics and Automation Magazine, pages 32-38, 1996.

[11] R. Balasubramanian and A. M. Dollar. A comparison of workspace and force capabilities between classes of underactuated mechanisms. In Proc. of the IEEE Internat. Conf. on Robotics and Automation, pages 3489-3496, 2011.

[12] H. Hanafusa and H. Asada. Robot Motion: Planning and Control, chapter Stable prehension by a robot hand with elastic fingers, pages 323-336. MIT Press Cambridge, 1982.

[13] R. Balasubramanian, J. T. Belter, and A. M. Dollar. External disturbances and coupling mechanisms in underactuated hands. In Proc. of ASME Internat. Design Engineering Technical Conf and Computers and Information in Engineering Conf., 2010.

[14] R. Balasubramanian, L. Xu, P. Brook, J. R. Smith, and $\mathrm{Y}$.Matsuoka. Human-guided grasp measures improve grasp robustness on physical robot. In Proc. IEEE Internat. Conf. on Robotics and Automat, pages 2294-2301, 2010.

[15] K. B. Shimoga. Robot grasp synthesis algorithms: A survey. Internat. J Robotics Res, 1996. DOI: $10.1177 / 027836499601500302$. 